\title{
Dynamic Band Allocation Method of XGS-PON System Using Time Sliding Window Traffic Prediction
}

\author{
Man Soo $\operatorname{Han}^{1}$ \\ ${ }^{1}$ Professor, Information and Communications Engineering Department, Mokpo National University, \\ Republic of Korea, mshan@mokpo.ac.kr
}

\begin{abstract}
The dynamic bandwidth allocation (DBA) algorithm has the greatest impact on the system performance in a 10-Gbps-capable symmetric passive optical network (XGS-PON). Most DBA algorithms perform DBA using only transmission requests sent from optical network units (ONUs) to optical line termination (OLT). In this paper, a new method to improve the performance by predicting the packet arrival rate of ONU and by using the predicted value and the transmission request of ONU at the same time in DBA is introduced. After the first dynamic bandwidth allocation with the request, the second dynamic bandwidth allocation is performed with the remaining bandwidth and predicted packet length. A new prediction method is proposed by applying the time sliding window method to the packet arrival rate prediction. In the proposed method, instead of predicting the packet arrival rate whenever one packet arrives, the packet arrival rate is predicted in unit time. The computer simulation showed that the proposed method performs better in packet mean delay than a method without packet prediction and a method using aggressive packet prediction. In addition, the length of the sliding window that optimizes the packet arrival rate prediction performance under burst traffic conditions is determined through simulation.
\end{abstract}

Keywords: XGS-PON, Dynamic, Bandwidth, Allocation, Traffic, Prediction, Performance, Evaluation

\section{Introduction}

10-Gbps-capable symmetric passive optical network (XGS-PON) system consists of one optical line termination (OLT) and multiple optical network units (ONU)[1][2]. For providing quality of service (QoS), multiple buffers are used for each service class in an ONU. When sending packets from an OLT to an ONU, a broadcasting method is used, but when sending packets from an ONU to an OLT, only one ONU can send packets to the OLT at a time. The OLT allocates a transmission time to ONUs so that two or more ONUs do not send packets to the OLT at the same time. The dynamic bandwidth allocation (DBA) is that the OLT allocates the transmission time to ONUs. Since the DBA determines the order in which ONUs transmit packets upstream, it has the greatest influence on the XGS-PON system performance. In the XGS-PON system, all operations are synchronized in $125 \mu$ s time unit, and DBA operation must be completed within $125 \mu$ s. Therefore, DBA operation should be as simple as possible.

Although many studies on DBA methods have been conducted[3-8], most of them decided the transmission time based on the request transmitted from ONUs. In [3], a pipelined DBA method was introduced to reduce power consumption. The DBA method of the time and wavelength division multiplexing (TWDM) PON system using multiple optical wavelengths for packet transmission was introduced in [4]. In [5], the DBA method of the 10-Gbps-capable passive optical network (XG-PON)

Received: July 11, 2021; $1^{\text {st }}$ Review Result: August 28, 2021; $2^{\text {nd }}$ Review Result: October 14, 2021

Accepted: November 30, 2021 
system was introduced. Service classes share one service pool, improving DBA performance. In [6], a DBA method was introduced for XG-PON system where ONU does not send a request to OLT. In [7], the DBA method of a system with multiple uplink channels was introduced. A DBA method was introduced for a next generation Ethernet passive optical network (NG-EPON) in [8].

All methods except [6] have limitations in improving upstream transmission performance because DBA is performed using only the request transmitted from ONU. [6] used a method of predicting traffic by measuring the usage of uplink bandwidth, but the DBA did not use the request transmitted from ONU. Since the physical maximum distance between ONU and OLT is $20 \mathrm{~km}$, it takes time for DBA result of OLT to be delivered to ONU, and new packets can arrive at ONU during this time. If the DBA uses the information on this newly arrived packet, the uplink transmission performance can be improved. At the time DBA is executed, the packets arriving in the future are not known, so a method of predicting packet arrival must be used.

Among the methods for predicting the arrival rate of packets, the time sliding window (TSW) prediction method [9] updates the average packet arrival rate for a predetermined time whenever one packet arrives. In the XGS-PON system, all operations are performed in $125 \mu$ s time unit, and the ONU request is also generated once during this $125 \mu$ s time unit. In order to apply the TSW method to the XGS-PON system, the update of the average packet arrival rate must be performed in every unit time not in every packet arrival.

In this paper, a new immediate allocation with time sliding window estimation (IATE) method is proposed for DBA using the modified TSW prediction method which predicts the amount of arrival packets arriving in the next unit time. In the proposed method, the first DBA is performed with only the current buffer length, and then the second DBA is performed with the remaining bandwidth and the predicted packet arrival rate. Using simulations, the performance of the proposed method is compared with the method without packet prediction and the method of predicting the packet arrival rate in the next unit time as the packet arrival rate in the current unit time. The proposed method shows better mean delay performance in higher service classes than other methods. Also, the optimal value for the window length used for traffic prediction is obtained through simulations.

\section{Dynamic Bandwidth Allocation}

The XGS-PON system consists of one OLT and N ONUs. To support multiple service classes, an ONU has a buffer for each service class. The service class is called traffic-container (T-CONT) type in the XGS-PON system. In this paper, only T-CONT types 2, 3, and 4 are considered. T-CONT type 2 is assured traffic in which transmission bandwidth allocation is guaranteed, and T-CONT type 3 is nonassured traffic in which transmission bandwidth allocation is not guaranteed. T-CONT type 4 is best effort traffic. [Fig. 1] illustrates an XGS-PON system. $R_{N}$ is the uplink bandwidth of the ONU. $R_{U}$ is the ONU input bandwidth. Buffer $\mathrm{q}_{\mathrm{ij}}$ of ONU indicates a buffer in which packets of T-CONT type $j$ in ONU $i$ are stored.

When transmitting a packet downlink from OLT to ONU, the packet is transmitted in a broadcasting manner through a splitter. The ONU receives a downlink packet and discards the packet if the packet destination does not match to the ONU. The uplink bandwidth $R_{N}$ is shared by all ONUs when a packet is transmitted from an ONU to the OLT. So, the uplink bandwidth is used by each ONU in a time division manner. Therefore, only one ONU can use the uplink bandwidth at a time. The OLT receives a request of each buffer from the ONUs, performs DBA to determine the uplink transmission time not to be duplicated for each ONU, and then delivers the result to each ONU.

The request sent from ONU to OLT is the sum of the lengths of packets stored in a buffer. The OLT determines and informs the command to send a request for each buffer $\mathrm{q}_{\mathrm{ij}}$ and the uplink transmission 
time to be used when sending the request. The buffer $\mathrm{q}_{\mathrm{ij}}$, which has received the command to send the request, informs the OLT of the total length of the packets stored in it. The more frequently buffer $\mathrm{q}_{\mathrm{ij}}$ sends requests, the better the OLT can understand the status of each buffer. However, since the uplink bandwidth is used when sending the request message, if the request message is frequently sent, the uplink bandwidth may be wasted. In this paper, it is assumed that the request sending period is different for each service class.

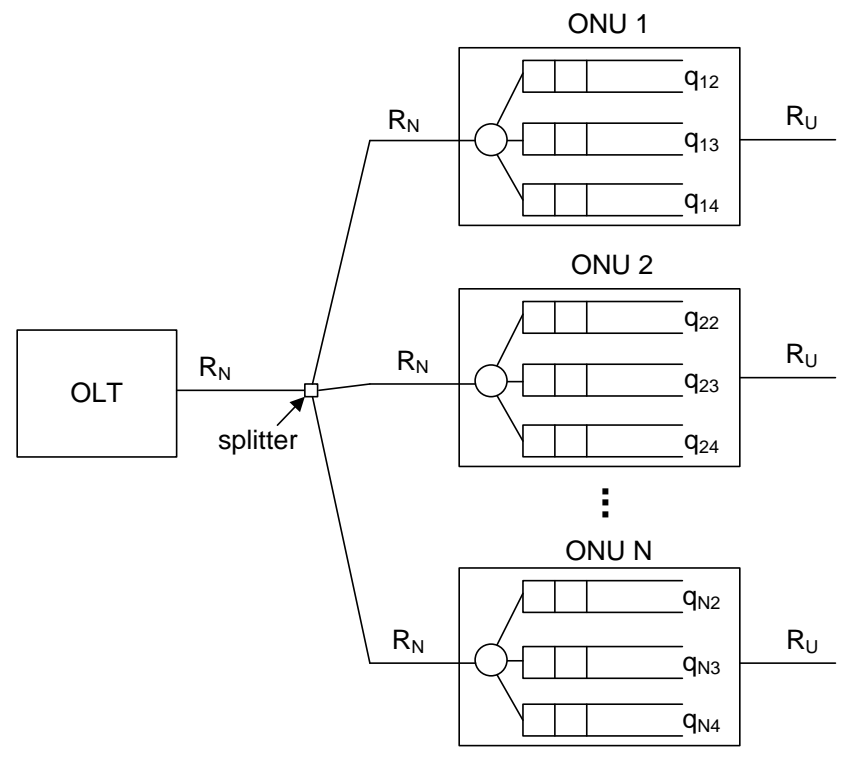

[Fig. 1] Block Diagram of XGS-PON System

All operations of XGS-PON are synchronized with unit time. In the XGS-PON system, the unit time is fixed at $125 \mu \mathrm{s}$. OLT executes DBA every unit time, stores the result in bandwidth map (BWmap), and transmits the BWmap to each ONU at the end of each unit time. Since all operations are synchronized with the unit time, the sum of the transmission time allocated by the DBA is also less than the unit time. The OLT can demand a request to an ONU. At this time, a separate uplink bandwidth for the request is allocated so that the ONU can send the request information to the OLT. This separate uplink bandwidth is called dynamic bandwidth allocation upstream (DBRu). In general, the buffer $\mathrm{q}_{\mathrm{ij}}$ sends the total length of the stored packets using DBRu. However, in this paper, it is assumed that the buffer $\mathrm{q}_{\mathrm{ij}}$ manages the amount of newly arrived packets for each unit time, and when $\mathrm{DBRu}$ is allocated, the newly arrived packet amount is transmitted to the OLT using the DBRu.

In this paper, the service interval of the buffer $\mathrm{q}_{\mathrm{ij}}$ is called $\mathrm{S}_{\mathrm{ij}}$. Also, the available amount of service that can be received during $S_{i j}$ is called $V_{i j}$. Also, the OLT stores the buffer length of $q_{i j}$, and the buffer length of the buffer $\mathrm{q}_{\mathrm{ij}}$ at unit time $\mathrm{t}$ is called $\mathrm{n}_{\mathrm{ij}}(\mathrm{t})$. Furthermore, the sum of the lengths of packets newly arrived at $\mathrm{q}_{\mathrm{ij}}$ during unit time $t$ is called $\mathrm{a}_{\mathrm{ij}}(\mathrm{t})$. Let the grant $\mathrm{g}_{\mathrm{ij}}(\mathrm{t})$ be the transmission amount that the OLT allows to the buffer $\mathrm{q}_{\mathrm{ij}}$ at the unit time $\mathrm{t}$, then the buffer length of the buffer $\mathrm{q}_{\mathrm{ij}}$ at the next unit time $\mathrm{t}+1$ is as follows.

$$
\mathrm{n}_{\mathrm{ij}}(\mathrm{t}+1)=\mathrm{n}_{\mathrm{ij}}(\mathrm{t})+\mathrm{a}_{\mathrm{ij}}(\mathrm{t})-\mathrm{g}_{\mathrm{ij}}(\mathrm{t})
$$

On the other hand, it takes time when the BWmap in which the DBA result is stored is transmitted from the OLT to each ONU, and if the distance between the OLT and the ONU is $20 \mathrm{~km}$, the time is $100 \mu \mathrm{s}$. The ONU needs about $30 \mu$ s preparation time after receiving the BWmap. Therefore, when the 
grant $g_{i j}(t)$ arrives at $\mathrm{q}_{\mathrm{ij}}$, more than one unit time has already elapsed, so there are new packets $\mathrm{a}_{\mathrm{ij}}(\mathrm{t}+1)$ in $\mathrm{q}_{\mathrm{ij}}$. Since the new packet length $\mathrm{a}_{\mathrm{ij}}(\mathrm{t}+1)$ cannot be known at unit time $\mathrm{t}$, it cannot be directly reflected in DBA at unit time $t$. In this paper, a new method is proposed for predicting the new arrival packet length and using this predicted packet length for DBA.

In this paper, the TSW prediction method introduced in [9] is modified. The TSW method is a method of calculating the average arrival rate of the next packet whenever a packet arrives. The TSW is known to predict packet arrival rates well in burst traffic conditions[10]. [Fig. 2] shows the TSW algorithm shown in [9].

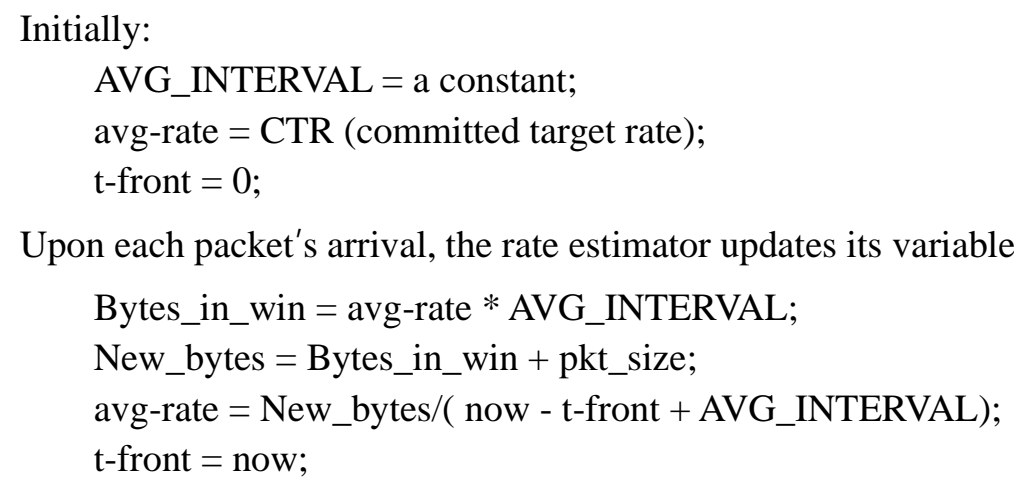

[Fig. 2] TSW Algorithm

In [Fig. 2], "now" is the arrival time of the current packet, "pkt_size" is the length of the arrived packet, "avg-rate" is the calculated average arrival rate, and "AVG_INTERVAL" is the length of the window that stores the arrival history.

Since the XGS-PON system is synchronized with a unit time of $125 \mu \mathrm{s}$, the TSW method is modified to calculate the average amount of arrival packets in unit time. Let $e_{i j}(t)$ be the average amount of arrived packets of the buffer $\mathrm{q}_{\mathrm{ij}}$ at unit time $\mathrm{t}$. Then at unit time $\mathrm{t}+1$, we have

$$
e_{i j}(\mathrm{t}+1)=\frac{\mathrm{e}_{i j}(\mathrm{t}) \times \text { AVG_INTERVAL }+\mathrm{a}_{i j}(\mathrm{t})}{1+\text { AVG_INTERVAL }}
$$

where AVG_INTERVAL has a unit of $125 \mu \mathrm{s}$. That is, if AVG_INTERVAL $=10$, it means $10 \mathrm{x}$ $125 \mu$ s. Also, it is assumed that $\mathrm{e}_{\mathrm{ij}}(0)=0$, which is the initial value of $\mathrm{e}_{\mathrm{ij}}(\mathrm{t})$.

In this paper, DBA is first executed using the buffer length $n_{i j}(t)$, and then DBA is additionally executed using the remaining service amount $\mathrm{V}_{\mathrm{ij}}$ and $\mathrm{e}_{\mathrm{ij}}(\mathrm{t}+1)$. If the total upstream bandwidth of the XGS-PON system is $F$, the first stage DBA algorithm using the buffer length $n_{i j}(t)$ is shown in [Fig. 3].

$$
\begin{aligned}
& \text { 1: } \quad \mathrm{g}_{\mathrm{ij}}(\mathrm{t}) \leftarrow \min \left\{\mathrm{V}_{\mathrm{ij}}, \mathrm{n}_{\mathrm{ij}}(\mathrm{t}), \mathrm{F}\right\} \\
& \text { 2: } \quad \mathrm{V}_{\mathrm{ij}} \leftarrow \mathrm{V}_{\mathrm{ij}}-\mathrm{g}_{\mathrm{ij}}(\mathrm{t}) \\
& \text { 3: } \quad \mathrm{n}_{\mathrm{ij}}(\mathrm{t}) \leftarrow \mathrm{n}_{\mathrm{ij}}(\mathrm{t})-\mathrm{g}_{\mathrm{ij}}(\mathrm{t}) \\
& \text { 4: } \quad \mathrm{F} \leftarrow \mathrm{F}-\mathrm{g}_{\mathrm{ij}}(\mathrm{t})
\end{aligned}
$$

[Fig. 3] Pseudo Code of First Stage DBA

The grant $g_{\mathrm{ij}}(\mathrm{t})$ is determined as the minimum value among $\mathrm{V}_{\mathrm{ij}}, \mathrm{n}_{\mathrm{ij}}(\mathrm{t})$, and $\mathrm{F}$, and each $\mathrm{V}_{\mathrm{ij}}, \mathrm{n}_{\mathrm{ij}}(\mathrm{t})$, and $\mathrm{F}$ are reduced by the grant $\mathrm{g}_{\mathrm{ij}}(\mathrm{t})$. [Fig. 4] shows the DBA algorithm of the second stage using the 
predicted packet arrival rate $\mathrm{e}_{\mathrm{ij}}(\mathrm{t}+1)$.

$\begin{array}{ll}\text { 1: } & \mathrm{G} \leftarrow \min \left\{\mathrm{V}_{\mathrm{ij}}, \mathrm{e}_{\mathrm{ij}}(\mathrm{t}+1), \mathrm{F}\right\} \\ \text { 2: } & \mathrm{g}_{\mathrm{ij}}(\mathrm{t}) \leftarrow \mathrm{g}_{\mathrm{ij}}(\mathrm{t})+\mathrm{G} ; \\ \text { 3: } & \mathrm{V}_{\mathrm{ij}} \leftarrow \mathrm{V}_{\mathrm{ij}}-\mathrm{G} \\ \text { 4: } & \mathrm{F} \leftarrow \mathrm{F}-\mathrm{G}\end{array}$

[Fig. 4] Pseudo Code of Second Stage

In [Fig. 4], the variable $G$ means the additional grant. The variable $G$ is calculated as the minimum value of $V_{i j}, e_{i j}(t+1), F$. Then, $V_{i j}$ and $F$ are each reduced by $G$, and $G$ is added to the grant $g_{i j}(t)$ that is calculated in the first stage DBA.

Meanwhile, after DBA, timer $T_{i j}$ decreases by 1 , and if $T_{i j}=0, V_{i j}$ is recharged with $B_{i j}$ and $T_{i j}$ is recharged with $S_{i j}$. Here, $B_{i j}$ is the maximum service amount that buffer $\mathrm{q}_{\mathrm{ij}}$ can receive during $S_{\mathrm{ij}}$. The $\mathrm{V}_{\mathrm{ij}}$ recharge algorithm is shown in [Fig. 5].

$\begin{array}{ll}\text { 1: } & \mathrm{T}_{\mathrm{ij}} \leftarrow \mathrm{T}_{\mathrm{ij}}-1 \\ \text { 2: } & \text { if } \mathrm{T}_{\mathrm{ij}}=0 \text { then } \\ \text { 3: } & \quad \mathrm{V}_{\mathrm{ij}} \leftarrow \mathrm{B}_{\mathrm{ij}} \\ \text { 4: } & \quad \mathrm{T}_{\mathrm{ij}} \leftarrow \mathrm{S}_{\mathrm{ij}} \\ \text { 5: } & \text { end if }\end{array}$

[Fig. 5] Pseudo Code of Recharge Stage

In this paper, during the second stage DBA, additional transmission bandwidth is allocated using the remaining $\mathrm{V}_{\mathrm{ij}}$ after performing the first stage DBA. If $\mathrm{S}_{\mathrm{ij}} \geq 2$, bandwidth loss may occur in the DBA of the next unit time due to the second stage DBA in the current unit time. For example, when $S_{i j}=2$ at unit time $t$, suppose that $e_{i j}(t+1)$ consumes all $V_{i j}$ in the second stage DBA. Then, even if $n_{i j}(t+1)>0$ in the first stage DBA at unit time $t+1$, we have $g_{i j}(t+1)=0$ since $V_{i j}=0$. The value $e_{i j}(t+1)$ cannot be accurate since it is a predicted value, resulting in bandwidth wastage. In order to minimize such bandwidth waste, it is assumed that $S_{\mathrm{ij}}$ of all T-CONT types 2 and 3 is $S_{\mathrm{ij}}=1$. Also, since T-CONT type 4 is best-effort traffic, the buffer length is not predicted, and the second stage DBA is not executed for T-CONT type 4.

[Fig. 6] is an example of the proposed method.

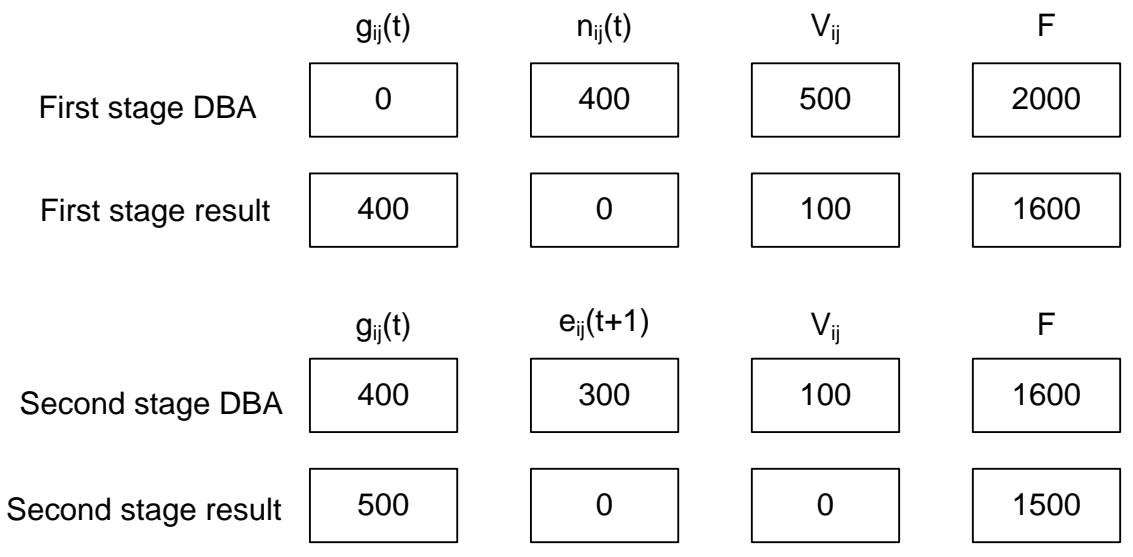

[Fig. 6] Example of the Proposed Method

In [Fig. 6], since $n_{i j}(t)=400, V_{i j}=500, F=2000$ in the first stage DBA, we get $g_{i j}(t)=\min \left\{n_{i j}(t)\right.$, 
$\left.\mathrm{V}_{\mathrm{ij}}, \mathrm{F}\right\}=400$. Then, we have $\mathrm{V}_{\mathrm{ij}}=\mathrm{V}_{\mathrm{ij}}-\mathrm{g}_{\mathrm{ij}}(\mathrm{t})=100, \mathrm{~F}=\mathrm{F}-\mathrm{g}_{\mathrm{ij}}(\mathrm{t})=1600$, and $\mathrm{n}_{\mathrm{ij}}(\mathrm{t})=\mathrm{n}_{\mathrm{ij}}(\mathrm{t})-\mathrm{g}_{\mathrm{ij}}(\mathrm{t})=0$. Assuming that $\mathrm{e}_{\mathrm{ij}}(\mathrm{t}+1)=300$ in the second stage DBA, $\mathrm{G}=\min \left\{\mathrm{e}_{\mathrm{ij}}(\mathrm{t}+1), \mathrm{V}_{\mathrm{ij}}, \mathrm{F}\right\}=100$, so $\mathrm{g}_{\mathrm{ij}}(\mathrm{t})=\mathrm{g}_{\mathrm{ij}}(\mathrm{t})$ $+100=500$. Therefore, the proposed algorithm allocates $g_{i j}(t)=500$ for the grant of buffer $\mathrm{q}_{\mathrm{ij}}$.

\section{Performance Evaluation}

In this section, the performance of the proposed method is evaluated. Assume that the total number of ONUs $\mathrm{N}=16$ and the uplink bandwidth $\mathrm{R}_{\mathrm{N}}=10 \mathrm{Gbps}$. The maximum user input bandwidth of each ONU is $R_{U}=1$ Gbps. In addition, the distance between OLT and ONU is $20 \mathrm{~km} \mathrm{[3][5][6]} \mathrm{and} \mathrm{the} \mathrm{size}$ of each $\mathrm{q}_{\mathrm{ij}}$ is 2 Mbyte. Also, in the case of T-CONT types 2 and 3, $\mathrm{S}_{\mathrm{ij}}=1$, and in the case of T-CONT type $4, \mathrm{~S}_{\mathrm{ij}}=5$. For T-CONT types 2 and $3, \mathrm{~B}_{\mathrm{ij}}=4732$ bytes, and for T-CONT type $4, \mathrm{~B}_{\mathrm{ij}}=48600$ bytes. Therefore, the bandwidth is $302.8 \mathrm{Mbps}$ for T-CONT 2 and 3 and $622 \mathrm{Mbps}$ for T-CONT type 4. Also, we use AVG_INTERVAL $=4$.

For comparison with other methods, first, the IATE performance is compared with that of the immediate allocation with colorless grant (IACG) method in [3]. At this time, the colorless grant part that does not conform to the XGS-PON standard in the IACG is not used. As the second comparison method, a commonly used traffic prediction method is used. In the second method, $\mathrm{e}_{\mathrm{ij}}(\mathrm{t}+1)=\mathrm{a}_{\mathrm{ij}}(\mathrm{t})$ is applied by assuming that the traffic arrival rates in the current and next unit times are the same. The second method is called the immediate allocation with aggressive estimation (IAAE) method.

The input traffic used in the simulation uses the self-similar traffic of [3]. Each ONU receives packets from an on-off traffic generation process that follows the Pareto distribution. For the on and off processes, the shape variables are 1.4 and 1.2, respectively. In addition, the packet size distribution uses a tri-modal distribution. The occurrence rates are 60\%,20\%, and 20\% for packet lengths of 64, 500, and 1500 bytes, respectively[3]. The ratio of traffic for each T-CONT type assumes balanced traffic. Therefore, the probability that an arrival packet to the ONU is input to the buffer for each TCONT type is the same. We wrote the simulation program by referring to the XGS-PON standard [2] and self-similar traffic generation method in [11] using the C++ language. The performance of each method was evaluated for the ONU input traffic load between 0.1 to 0.99 . Each simulation was carried out until the number of packets transmitted from ONUs to OLT reached $10^{9}$.

The mean delay of packets is calculated using the sample mean calculation in [12]. Let the variable $n$ be the number of packets transmitted from ONUs to OLT. In this paper, $n=10^{9}$. Let $x_{i}$ be the delay of the i-th packet transmitted from ONUs to OLT. Let $\mathrm{a}_{\mathrm{i}}$ be the time at which packet $i$ arrives at the ONU and $\mathrm{d}_{\mathrm{i}}$ be the time at which packet $i$ arrived at the OLT after it was sent from the ONU. Then, $\mathrm{x}_{\mathrm{i}}=$ $\mathrm{d}_{\mathrm{i}}-\mathrm{a}_{\mathrm{i}}$, and the mean delay $m d$ is as follows.

$$
m d=\frac{1}{\mathrm{n}} \sum_{i=1}^{n} x_{i} .
$$

The packet delay variance is calculated using the sample variance calculation in [12]. The packet delay variance $\mathrm{s}^{2}$ is as follows.

$$
\mathrm{s}^{2}=\frac{1}{\mathrm{n}} \sum_{i=1}^{n}\left(x_{i}-m d\right)^{2} .
$$

[Fig. 7] and [Fig. 8] depict the mean packet delays for T-CONT type 2 and the mean packet delays for T-CONT types 3 and 4, respectively. [Fig. 9] and [Fig. 10] show the packet delay variances for TCONT types 2, 3 and 4 . As shown in each figure, the proposed IATE method is superior in mean delays of T-CONT types 2 and 3 compared to other methods. 
In the T-CONT type 2 mean delay, IATE was improved from a minimum of $0.3 \%$ (load 0.99 ) to a maximum of $10.7 \%$ (load 0.4) compared to IAAE. It was improved from a minimum of $0.9 \%$ (load 0.99 ) to a maximum of $31.7 \%$ (load 0.1) compared to IACG. In the mean delay performance of TCONT type 3, IATE was improved from a minimum of $0.3 \%$ (load 0.99 ) to a maximum of $10.6 \%$ (load 0.4) compared to IAAE. It was improved from a minimum of $1.0 \%$ (load 0.99 ) to a maximum of $31.6 \%$ (load 0.1) compared to IACG. However, in the T-CONT type 4 mean delay, IATE deteriorated up to $-15.8 \%$ (load 0.6) compared to IAAE, and up to $-20.3 \%$ (load 0.6) worse than IACG.

The proposed IATE method was better in the mean delay of T-CONT types 2 and 3 than other methods. The reason is that the DBA additionally grants a bandwidth allocation to the predicted amount of traffic in the proposed method. As a result, since T-CONT types 2 and 3 use more uplink bandwidth than other methods, the bandwidth used by T-CONT type 4 is reduced. Therefore, in IATE, the mean delay of T-CONT type 4 was worse than that of other methods. However, since T-CONT type 4 is best-effort traffic, it is reasonable that T-CONT types 2 and 3, which have higher service priorities, use more uplink bandwidth.

If the DBA does not use traffic prediction, the ONU must send a transmission request to the OLT in order for the packet to get a grant. Then, the DBA must grant the transmission for the request, and the DBA result must be sent back to ONU. However, if the DBA uses traffic prediction, some packets can receive transmission grant immediately without sending the transmission request. This is the reason why the difference in the mean delay between packets becomes large in the proposed method. Therefore, the delay variance is larger than other methods in the proposed method.

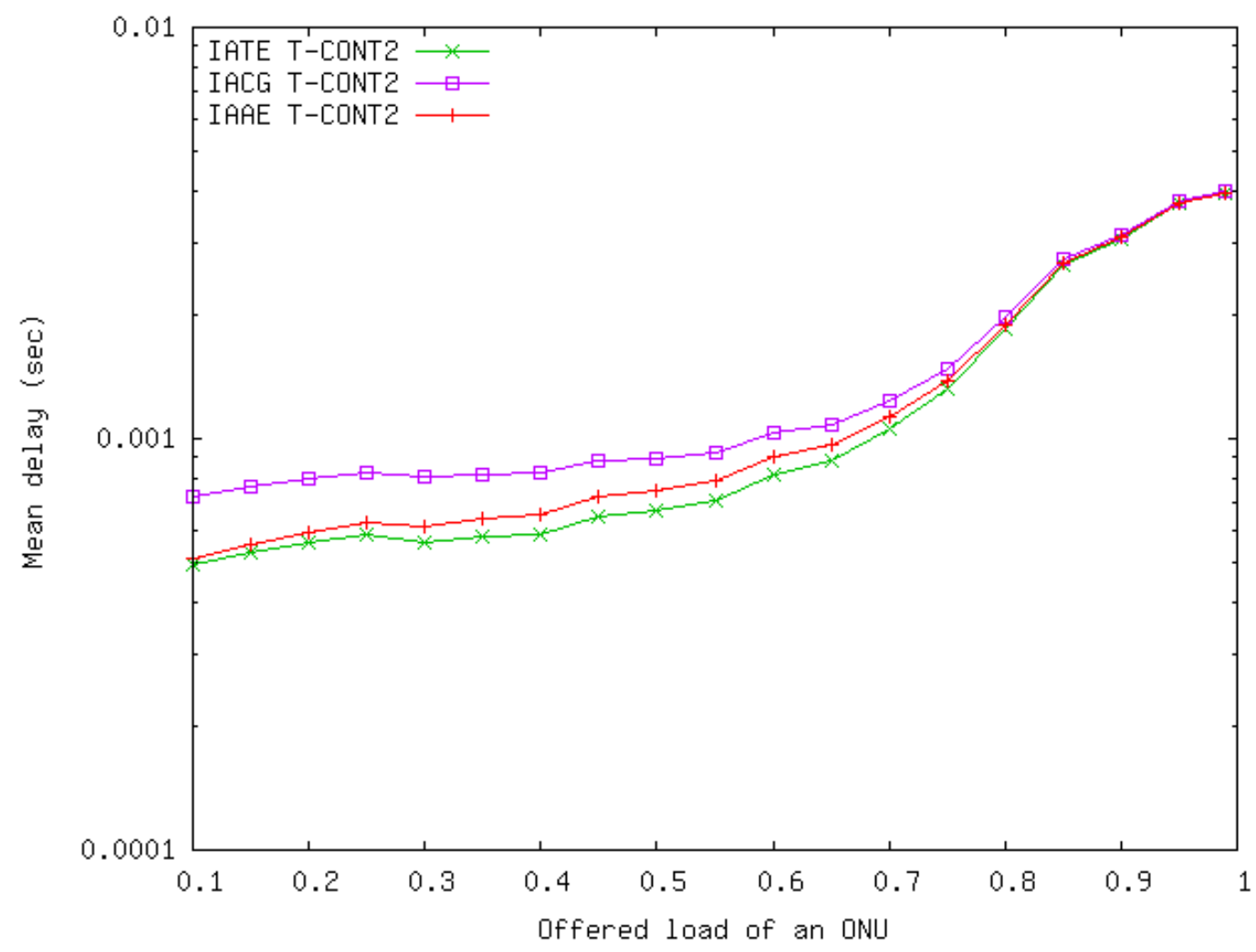

[Fig. 7] Mean Packet Delay of T-CONT Type 2 


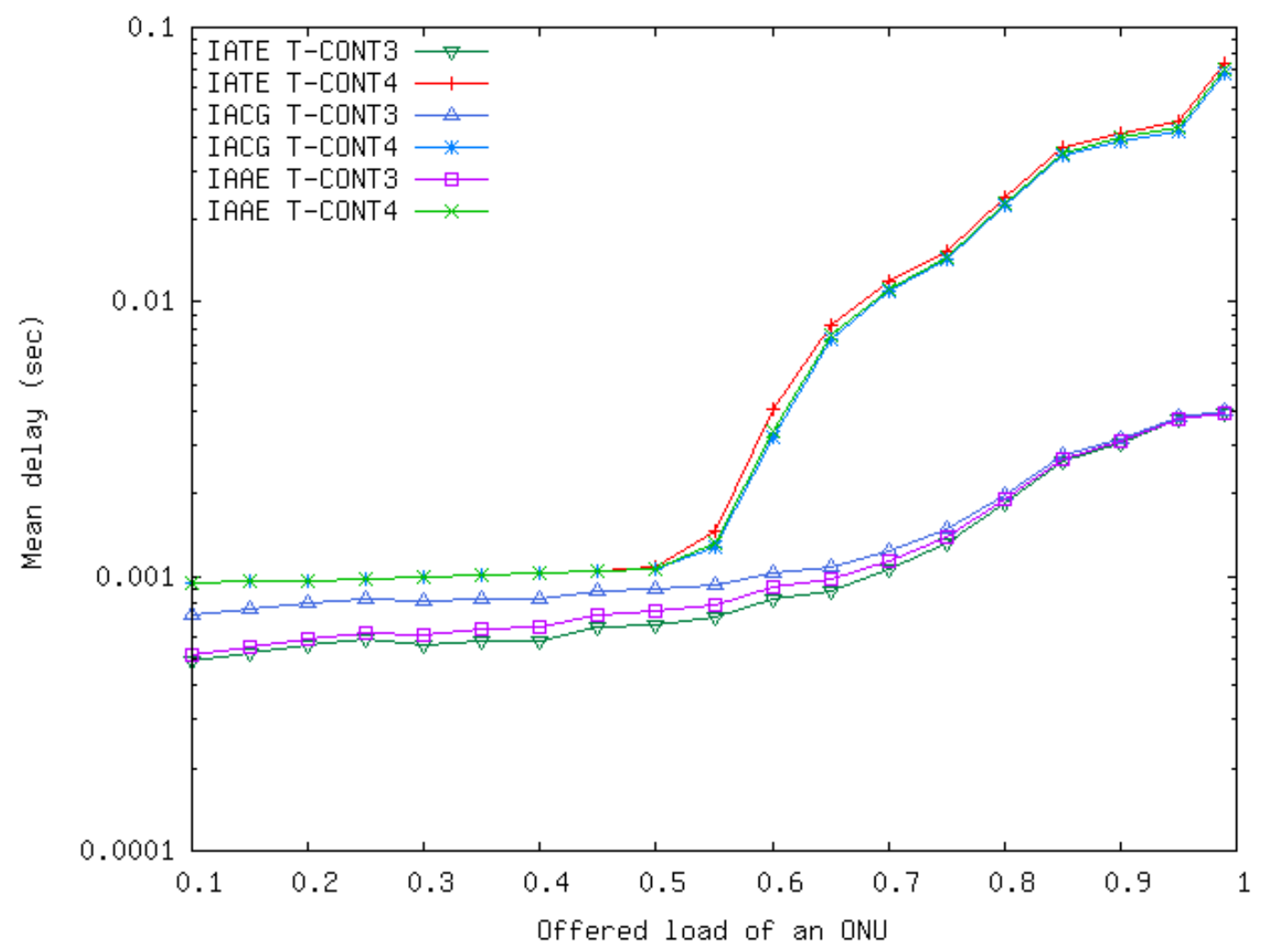

[Fig. 8] Mean Packet Delay of T-CONT Types 3 and 4

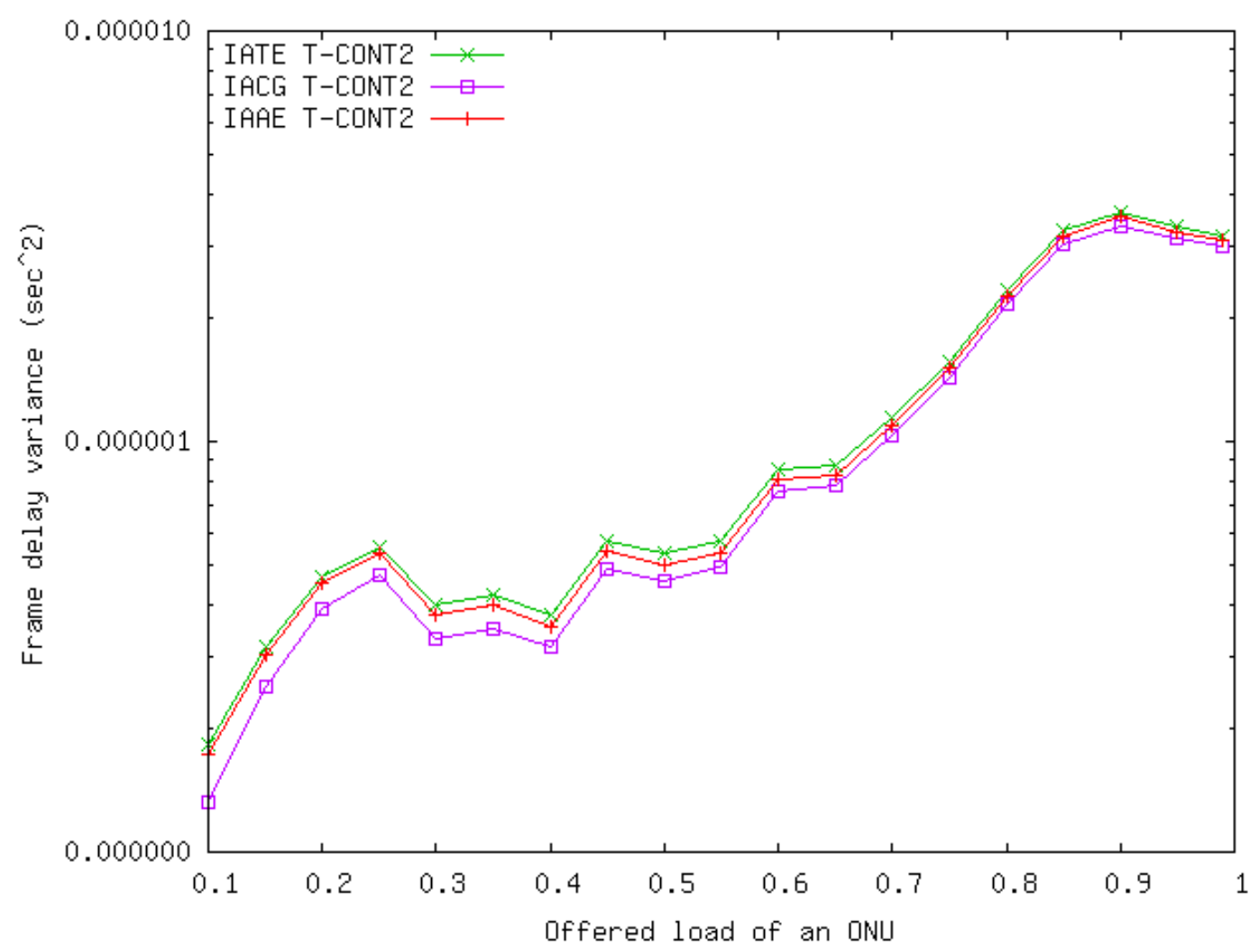

[Fig. 9] Packet Delay Variance of T-CONT Type 2 


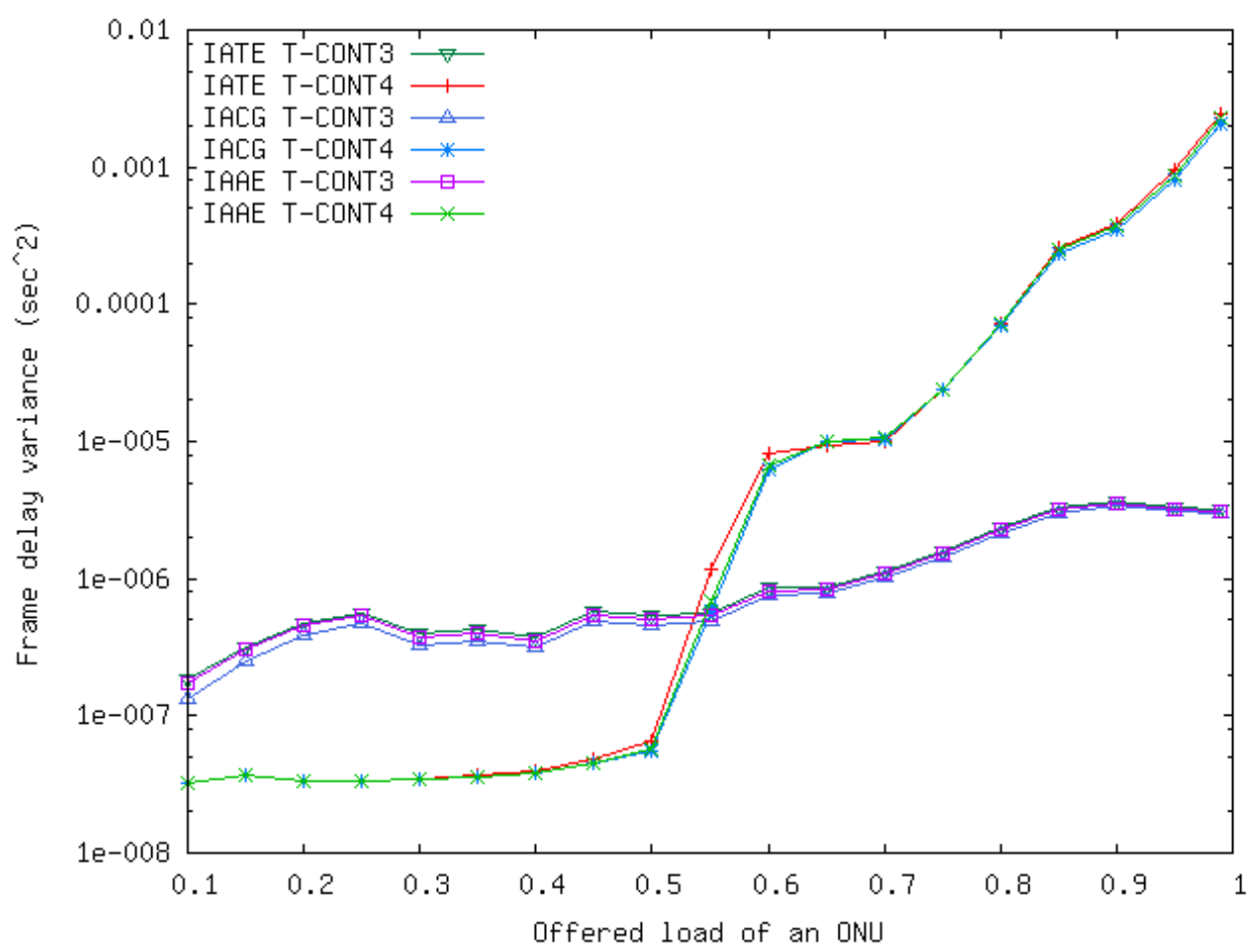

[Fig. 10] Packet Delay Variance of T-CONT Types 3 and 4

In the previous simulation, AVG_INTERVAL $=4$ was assumed. From now on, the traffic prediction performance will be examined according to the AVG_INTERVAL value. [Fig. 11] shows the mean packet delay of T-CONT type 2 when the AVG_INTERVAL values are 4, 64, and 128. As shown in [Fig. 11], performance is best when AVG_INTERVAL=128 and worst when AVG_INTERVAL=4, with a difference of up to $10 \%$. However, when AVG_INTERVAL $=64$, there is little difference from AVG_INTERVAL=128, so the AVG_INTERVAL=64 can be considered as an appropriate value.

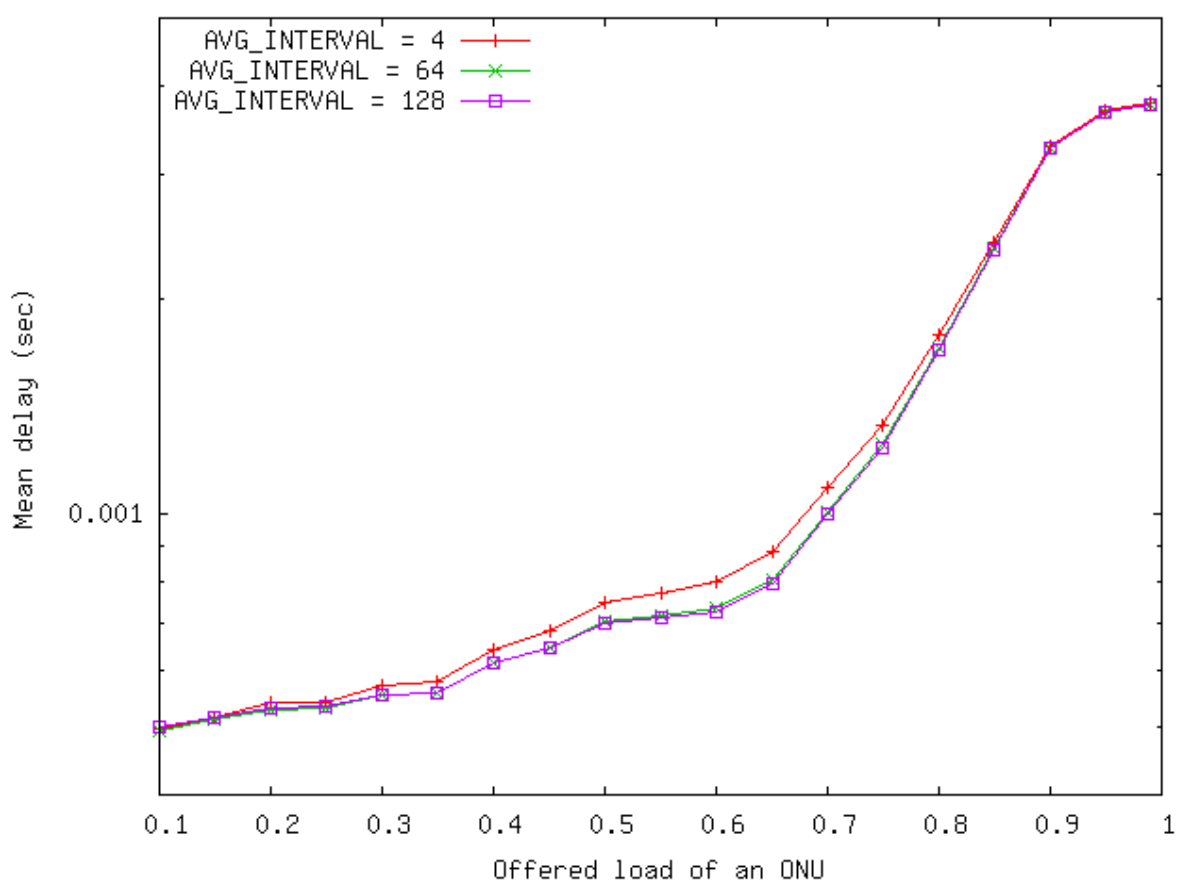

[Fig. 11] Mean Packet Delay of T-CONT Type 2 


\section{Conclusions}

In this paper, the researchers proposed the IATE method, which is a new method for DBA of XGSPON using the modified TSW prediction method to predict the amount of arrival packets in the next unit time. First, DBA is performed with only the current buffer length, and then additional DBA is performed with the remaining bandwidth and predicted packet arrival rate. The IATE performance was compared with the performance of a method without packet prediction and with the performance of a method with aggressive packet prediction. It was verified that the proposed method was superior in mean delay of the higher service classes compared to these two methods under the same input traffic. In this paper, the TSW method, which belongs to a simple method for traffic prediction, is modified and used. DBA performance can be further improved if more sophisticated traffic prediction methods are used. As a future study, a traffic prediction method using a machine learning method is considered.

\section{References}

[1] ITU-T Rec. G.989, 40-Gigabit-capable passive optical networks (NG-PON2): Definitions, abbreviations and acronyms, International Telecommunication Union, (2015)

[2] ITU-T Rec. G.9807.1, 10-Gigabit-capable symmetric passive optical network (XGS-PON), International Telecommunication Union, (2016)

[3] M. S. Han, Pipelined Architecture of Dynamic Bandwidth Allocation for Energy Efficiency in XG-PON, Contemporary Engineering Sciences, (2014), Vol.7, No.24, pp.1355-1362, DOI: http://dx.doi.org/10.12988/ ces.2014.49167

[4] A. Dixit, B. Lannoo, D. Colle, M. Pickavert, P. Demeester, Dynamic Bandwidth Allocation with Optimal Wavelength Switching in TWDM-PONs, 2013 15th International Conference on Transparent Optical Networks (ICTON), (2013), June 23-27; Cartagena, Spain, DOI: https://doi.org/10.1109/icton.2013.6602854

[5] A. Gupta, C. M. Machuca, A. Dixit, XGPON Statistical Multiplexing Analysis with Heterogeneous Users, 2018 20th International Conference on Transparent Optical Networks (ICTON), (2018), July 1-5; Bucharest, Romania, DOI: https://doi.org/10.1109/icton.2018.8473786

[6] M. S. Han, Performance Evaluation of Dynamic Bandwidth Allocation Algorithm for XG-PON Using Traffic Monitoring and Intra-Scheduling, International Journal of Software Engineering and its Applications, (2015), Vol.9, No.8, pp. 207-216, DOI: https://doi.org/10.14257/astl.2015.108.15

[7] Y. Yang, M. Zhang, D. Wang, J. Liu, Z. Ghassemlooy, DBA with consideration of energy, delay and BW for community WDM-OFDM PON, 2015 Opto-Electronics and Communications Conference (OECC), (2015), June 28July 2; Shanghai, China, DOI: https://doi.org/10.1109/oecc.2015.7340186

[8] S. B. Hussain, W. Hu, C. Li, Fair DWBA for WA-PON based NG-EPON (100G-EPON) to mitigate frame resequencing problem, 2017 Opto-Electronics and Communications Conference (OECC) and Photonics Global Conference (PGC), (2017), July 31-August 4; Singapore, DOI: https://doi.org/10.1109 /oecc.2017.8114817

[9] W. Fang, N. Seddigh, B. Nandy, A Time Sliding Window Three Colour Marker, Network Working Group, (2020), RFC2859, https://www.rfc-editor.org/rfc/rfc2859.html

[10] K. Salah, F. Haidari, On the Performance of a Simple Packet Rate Estimator, 2008 IEEE/ACS International Conference on Computer Systems and Applications, (2008), March 31-April 4; Doha, Qatar, DOI: https://doi.org/10.1109/aiccsa.2008.4493563

[11] G. Kramer, Ethernet Passive Optical Networks, McGraw-Hill Professional, (2005)

[12] D. B. Thomas and W. Luk, Estimation of Sample Mean and Variance for Monte-Carlo Simulations, 2008 International Conference on Field-Programmable Technology, (2008), December 8-10; Taipei, Taiwan, DOI: https://doi.org/10.1109/fpt.2008.4762370 